\title{
Molecular identification of pathogenicity genes and ERIC types in Vibrio cholerae 01 epidemic strains from Mozambique
}

\author{
E. FOLGOSA ${ }^{1}$, S. MASTRANDREA ${ }^{2}$, P. CAPPUCCINELLI $^{3}$, S. UZZAU ${ }^{3}$, \\ P. RAPPELLI ${ }^{3}$, M. J. BRIAN ${ }^{4}$ AND M. M. COLOMBO ${ }^{5 *}$ \\ ${ }^{1}$ Department of Microbiology, Faculty of Medicine, University E. Mondlane, Mozambique \\ ${ }^{2}$ Clinic of Infectious Diseases, Faculty of Medicine, University of Sassari, Italy \\ ${ }^{3}$ Department of Biomedical Sciences, Faculty of Medicine, University of Sassari, Italy \\ ${ }^{4}$ Maputo Central Hospital, Mozambique \\ ${ }^{5}$ Department of Cellular and Developmental Biology, Faculty of Sciences, University of Rome, Italy
}

(Accepted 13 March 2001)

\section{SUMMARY}

The phenotypic and genotypic profiles of the $V$. cholerae strains causing the Mozambican 1997-8 epidemic were characterized to provide a reference for comparison with other epidemic strains. A total of 75 strains of $V$. cholerae $\mathrm{O} 1$ isolated in different provinces, were analysed. Strains were characterized by PCR for detecting toxin genes $(\operatorname{ctx} \mathrm{A}$, zot and ace), virulence associated genes $(t c p \mathrm{~A}, n a n \mathrm{H}, h l y \mathrm{~A}$ and $t o x \mathrm{R})$ and ERIC sequences. All $V$. cholerae strains were serotype O1, Ogawa, biotype El Tor. MIC testing showed a high proportion of strains multi-resistant to drugs ( $100 \%$ to cotrimoxazole and $52 \%$ to tetracycline) and susceptibility to ciprofloxacin. The isolates contained two intact copies of the CTX genetic element and all other genes tested. PCR of restricted DNA revealed two ERIC types: the first in provincial isolates, also predominant in other African epidemic strains, and the second in Maputo isolates (the national capital).

\section{INTRODUCTION}

Since the seventh cholera pandemic reached Africa in the 1970s, epidemics have become recurrent and endemic cholera has been established in many regions. Vibrio cholerae $\mathrm{O} 1 \mathrm{El}$ Tor drug resistance also became a major health problem for many African countries.

In 1997 Africa suffered many outbreaks favoured by environmental factors (heavy rainfall and floods possibly related to El Niño phenomenon). More than 118000 cholera cases distributed among 25 African countries were reported (WHO, WER; 1998). Cholera epidemics affected all East Africa and spread to the Austral region. One of the most affected countries was

\footnotetext{
* Author for correspondence: DIP. Biologia Cellulane e dello Sviluppo, Sezione Scienze Microbiologiche, Facolta di Scienze M. F. N., Università di Roma "La Sapienza", Via dei Sardi 70, 00185, Roma, Italy.
}

Mozambique. The seventh cholera pandemic reached Mozambique in 1973 (WHO, WER; 1974). An epidemic phase ended in 1975, followed by an endemic phase up to 1985. Cholera re-emerged in 1989 in the northern province of Tete and reached Maputo (the national capital located in the south) in 1991 with more than 3600 cases being reported in the next 2 years. The number of cases was highest during the hot rainy season (December-May) [1]. The causative organism was $V$. cholerae O1, El Tor, serotype Inaba (with a few sporadic Ogawa), susceptible to tetracycline, sulfadiazine and chloramphenicol. Doxycycline (an analogue of tetracycline) was widely used for patient and contact treatment [2]. Cholera disappeared again in 1992.

The epidemic under study started in 1997 in Maputo and spread north to other provinces. About 41000 cases $(2 \cdot 7 \%$ mortality) were registered up to October 
1999 (WHO web site); sporadic cases and local outbreaks are still occurring in the country.

Molecular epidemiology is based mostly on chromosomal polymorphism [3,4] as an indicator of clonal relationships among different epidemic isolates. Variation in ribosomal RNA gene sequences [5-8], repetitive sequence elements in $V$. cholerae (VCR) [9], randomly amplified polymorphic DNA (RAPDPCR) [10] and enterobacterial repetitive intergenic consensus (ERIC) sequences [11-13] are currently used alongside variations in drug resistance patterns as epidemiological markers. ERIC are highly conserved sequences, present as multiple copies in the genome of bacteria, with no link to pathogenic genes. ERIC markers were chosen in this study since the PCR fingerprinting method is highly discriminatory in investigating clonal origin of epidemic strains and is the most rapid and reliable technique available [14].

Determining the potential virulence of isolates is an important first step in characterizing the genetic population structure of epidemic $V$. cholerae. Because many factors combine to determine $V$. cholerae pathogenicity, testing for the presence of at least the main virulence genes is required. The main pathogenic mechanism of $V$. cholerae $\mathrm{O} 1$ is the production of three enterotoxins. In addition to the heat-labile cholera toxin (CT), $V$. cholerae $\mathrm{O} 1$ produces the zonula occludens toxin (Zot) $[15,16]$ and the accessory cholera enterotoxin (Ace) [17]. The toxin genes (ctx, ace and zot) are clustered in a toxigenic cassette, named CTX, in a $4.5 \mathrm{kbp}$ region, flanked by directly repeated sequences (RS) of $2 \cdot 4-2.7 \mathrm{kbp}$ [18]. This toxigenic cassette is encoded by a filamentous bacteriophage (СТXФ) [19] able to infect the $V$. cholerae O1 cell by recognizing TcpA (toxin coregulated pilus) surface protein [20]. The resulting strain produces cholera toxin and causes disease. Other genes associated with virulence include $t c p \mathrm{~A}$, tox $\mathrm{R}$ (toxin regulator gene) [21], $\operatorname{nan} \mathrm{H}$ (neuraminidase, or NANase) and hlyA (haemolysin) [22]. TcpA is the major structural subunit of $V$. cholerae pilin of which toxin-coregulated pili are composed; and these are required for colonization of microvilli in the small bowel. $t c p \mathrm{~A}$ gene is coordinately expressed within a virulence regulon with DNA-binding and transmembrane regulatory protein ToxR [21]. Several environmental conditions can modulate the expression of tox $\mathrm{R}$ and its product is at the top of a regulatory cascade activating transcription of $c t x, t c p$ and other accessory virulence genes. $V$. cholerae produces a neuraminidase, which converts ganglio- sides to toxin receptors, enhancing the binding of CT and leading to greater fluid secretion. Genes encoding haemolysin are present in classical, El Tor and nonO1 strains of $V$. cholerae. Haemolysis of sheep erythrocytes was traditionally used to distinguish between El Tor and classical biotypes.

The aim of the study was to characterize the phenotypic and genotypic profile of $V$. cholerae strains isolated in Mozambique to obtain a better picture of the transmission of strains in Mozambique and in the region.

\section{MATERIALS AND METHODS}

\section{V. cholerae isolates and reference strains}

From August 1997 to June 1998 a total of $75 \mathrm{~V}$. cholerae epidemic strains were isolated by conventional methods (enrichment in Alkaline Peptone Water and subculture on TCBS agar) from hospitalized patients with clinical diagnosis of cholera (according to WHO guidelines). Strains and characterizations are listed in Table 1. Strains were identified by biochemical tests (API $20 \mathrm{E}$, bioMerieux) and specific antisera, tested for susceptibility to drugs and stored for further characterization.

$V$. cholerae O1 395, Ogawa serotype, and Escherichia coli HB 101 were respectively used as positive and negative control in PCR experiments. $V$. cholerae O1 Strains from African countries were used to compare ERIC types: Angola (VC663) (13), Rwanda (CNRVC940183, Institut Pasteur, Paris, France), Burundi (CNRVC940173, Institut Pasteur, Paris, France), Somalia (VCM26) (23), Swaziland (VCP2, Royal Min. of Health), Zimbabwe (VC4790A, Min. of Health). Additional reference strains were from Italy (VCBA8, Instituto di Igiene, University of Bari, 1994) and from India (O1 VC20 1992, O139 SG24 1992, O1 CO840 1995, O139 PG321 1998, National Institute of Cholera Enteric Disease, Calcutta).

\section{Susceptibility to antimicrobial agents}

After preliminary Kirby Bauer testing by disk diffusion on Mueller Hinton agar plates, minimum inhibitory concentrations (MICs) were determined for all 75 isolates with few omissions (see Table 1). MICs were measured by applying a bacterial suspension onto agar plates containing dilutions of the following drugs: ampicillin, chloramphenicol, ciprofloxacin, erythromycin, kanamycin, nalidixic acid, penicillin, 
Table 1. Phenotypic and molecular characterization of Vibrio cholerae isolates from 1997-8 cholera epidemic in Mozambique

\begin{tabular}{|c|c|c|c|c|c|c|c|c|c|c|c|c|}
\hline \multicolumn{3}{|c|}{ Isolate and place } & \multirow{2}{*}{$\frac{\mathrm{CTX}^{*}}{+}$} & \multirow{2}{*}{$\frac{\mathrm{ERIC}^{\dagger}}{5}$} & \multirow{2}{*}{$\frac{\mathrm{Amp}}{\mathrm{S} \ddagger}$} & \multirow{2}{*}{$\begin{array}{l}\text { Chl } \\
\mathrm{S}\end{array}$} & \multirow{2}{*}{$\begin{array}{l}\text { Ery } \\
\text { I }\end{array}$} & \multirow{2}{*}{$\begin{array}{l}\text { Kan } \\
\text { I }\end{array}$} & \multirow{2}{*}{$\begin{array}{l}\mathrm{Nal} \\
\mathrm{S}\end{array}$} & \multirow{2}{*}{$\begin{array}{l}\text { Pen } \\
S\end{array}$} & \multirow{2}{*}{$\frac{\text { Rif }}{\text { I }}$} & \multirow{2}{*}{$\frac{\text { Tet }}{\mathrm{I}}$} \\
\hline VCMP & 5556 & Maputo & & & & & & & & & & \\
\hline VCMP & 5559 & Maputo & - & - & $\mathrm{S}$ & I & I & I & $\mathrm{S}$ & $\mathrm{S}$ & I & $\mathrm{R}$ \\
\hline VCMP & 5560 & Maputo & - & - & $\mathrm{S}$ & I & I & I & $\mathrm{S}$ & $\mathrm{S}$ & I & $\mathrm{R}$ \\
\hline VCMP & 5594 & Maputo & + & 5 & $\mathrm{~S}$ & $\mathrm{~S}$ & I & I & $\mathrm{S}$ & $\mathrm{S}$ & I & I \\
\hline VCMP & 5622 & Maputo & - & - & $\mathrm{S}$ & $\mathrm{S}$ & I & I & $\mathrm{S}$ & $\mathrm{S}$ & I & I \\
\hline VCMP & 5623 & Maputo & - & - & $\mathrm{S}$ & $\mathrm{S}$ & I & I & $\mathrm{S}$ & $\mathrm{S}$ & I & I \\
\hline VCMP & 5632 & Maputo & - & - & $\mathrm{R}$ & $\mathrm{R}$ & I & I & $\mathrm{S}$ & $\mathrm{S}$ & I & $\mathrm{R}$ \\
\hline VCMP & 5633 & Maputo & - & - & $\mathrm{S}$ & $\mathrm{S}$ & I & I & $\mathrm{S}$ & $\mathrm{S}$ & I & $\mathrm{R}$ \\
\hline VCMP & 5635 & Maputo & - & - & $\mathrm{S}$ & $\mathrm{S}$ & I & I & $\mathrm{S}$ & $\mathrm{S}$ & I & I \\
\hline VCMP & 5663 & Maputo & - & - & $\mathrm{R}$ & $\mathrm{R}$ & $\mathrm{R}$ & $\mathrm{R}$ & $\mathrm{R}$ & $\mathrm{R}$ & $\mathrm{R}$ & $\mathrm{R}$ \\
\hline VCMP & 5666 & Maputo & + & 5 & $\mathrm{~S}$ & $\mathrm{~S}$ & I & I & $\mathrm{S}$ & $\mathrm{S}$ & I & $\mathrm{S}$ \\
\hline VCMP & 5669 & Maputo & - & - & $\mathrm{S}$ & $\mathrm{S}$ & I & I & $\mathrm{S}$ & $\mathrm{S}$ & I & $\mathrm{R}$ \\
\hline VCMP & 5673 & Maputo & - & - & $\mathrm{S}$ & $\mathrm{R}$ & I & I & $\mathrm{S}$ & $\mathrm{S}$ & I & $\mathrm{R}$ \\
\hline VCMP & 5738 & Maputo & - & - & $\mathrm{S}$ & $\mathrm{S}$ & I & I & $\mathrm{S}$ & $\mathrm{S}$ & I & $\mathrm{S}$ \\
\hline VCMP & 5742 & Maputo & - & - & $\mathrm{S}$ & $\mathrm{S}$ & I & I & $\mathrm{S}$ & $\mathrm{S}$ & I & I \\
\hline VCMP & 5767 & Maputo & - & - & $\mathrm{R}$ & $\mathrm{R}$ & $\mathrm{R}$ & I & $\mathrm{R}$ & $\mathrm{R}$ & I & I \\
\hline VCMP & 5768 & Maputo & - & - & $\mathrm{S}$ & $\mathrm{S}$ & I & I & $\mathrm{S}$ & $\mathrm{S}$ & I & I \\
\hline VCMP & 5806 & Maputo & + & 5 & $\mathrm{~S}$ & $\mathrm{~S}$ & I & $\mathrm{R}$ & $\mathrm{R}$ & $\mathrm{S}$ & I & $\mathrm{S}$ \\
\hline VCMP & 5807 & Maputo & - & - & $\mathrm{R}$ & $\mathrm{S}$ & I & $\mathrm{R}$ & $\mathrm{S}$ & $\mathrm{S}$ & I & I \\
\hline VCMP & 5834 & Maputo & + & 5 & - & $\mathrm{R}$ & $\mathrm{S}$ & - & $\mathrm{S}$ & - & - & $\mathrm{R}$ \\
\hline VCMP & 5818 & Maputo & - & - & $\mathrm{R}$ & $\mathrm{S}$ & $\mathrm{R}$ & $\mathrm{R}$ & $\mathrm{S}$ & $\mathrm{R}$ & $\mathrm{R}$ & $\mathrm{S}$ \\
\hline VCMP & 5842 & Maputo & - & - & $\mathrm{R}$ & $\mathrm{R}$ & I & $\mathrm{R}$ & $\mathrm{S}$ & $\mathrm{S}$ & I & $\mathrm{R}$ \\
\hline VCMP & 5843 & Maputo & - & - & $\mathrm{S}$ & $\mathrm{S}$ & I & $\mathrm{R}$ & $\mathrm{R}$ & $\mathrm{S}$ & I & I \\
\hline VCMP & 5844 & Maputo & - & - & $\mathrm{S}$ & $\mathrm{S}$ & I & $\mathrm{R}$ & $\mathrm{S}$ & $\mathrm{S}$ & I & I \\
\hline VCMP & 5847 & Maputo & + & 5 & - & $\mathrm{S}$ & $\mathrm{S}$ & - & $\mathrm{S}$ & - & - & $\mathrm{R}$ \\
\hline VCMP & 5952 & Maputo & - & - & $\mathrm{R}$ & $\mathrm{S}$ & $\mathrm{R}$ & $\mathrm{R}$ & $\mathrm{R}$ & $\mathrm{R}$ & $\mathrm{R}$ & I \\
\hline VCMP & 5953 & Maputo & - & - & $\mathrm{S}$ & $\mathrm{S}$ & I & $\mathrm{R}$ & $\mathrm{S}$ & $\mathrm{S}$ & I & I \\
\hline VCMP & 6035 & Maputo & - & - & $\mathrm{R}$ & $\mathrm{S}$ & I & $\mathrm{R}$ & $\mathrm{S}$ & $\mathrm{S}$ & I & I \\
\hline VCMP & 6037 & Maputo & - & - & $\mathrm{R}$ & $\mathrm{S}$ & I & $\mathrm{R}$ & $\mathrm{S}$ & $\mathrm{S}$ & I & I \\
\hline VCMP & 6047 & Maputo & - & - & $\mathrm{R}$ & $\mathrm{S}$ & I & $\mathrm{R}$ & $\mathrm{S}$ & $\mathrm{S}$ & I & I \\
\hline VCMP & 6049 & Maputo & + & 5 & - & $\mathrm{R}$ & $\mathrm{R}$ & - & I & - & - & $\mathrm{R}$ \\
\hline VCMP & 6075 & Maputo & - & - & $\mathrm{S}$ & $\mathrm{S}$ & I & $\mathrm{R}$ & $\mathrm{S}$ & $\mathrm{S}$ & I & I \\
\hline VCMP & 6079 & Maputo & - & - & $\mathrm{S}$ & $\mathrm{S}$ & I & $\mathrm{R}$ & $\mathrm{S}$ & $\mathrm{S}$ & I & I \\
\hline VCMP & 6125 & Maputo & - & - & $\mathrm{S}$ & $\mathrm{S}$ & I & $\mathrm{R}$ & $\mathrm{S}$ & $\mathrm{S}$ & I & I \\
\hline VCMP & 6127 & Maputo & + & 5 & $\mathrm{R}$ & $\mathrm{S}$ & $\mathrm{R}$ & $\mathrm{R}$ & $\mathrm{S}$ & $\mathrm{R}$ & $\mathrm{R}$ & I \\
\hline VCMP & 6147 & Maputo & - & - & $\mathrm{R}$ & $\mathrm{S}$ & $\mathrm{R}$ & $\mathrm{R}$ & $\mathrm{R}$ & $\mathrm{R}$ & I & $\mathrm{S}$ \\
\hline VCMP & 6149 & Maputo & - & - & $\mathrm{R}$ & $\mathrm{R}$ & I & $\mathrm{R}$ & $\mathrm{S}$ & $\mathrm{S}$ & I & $\mathrm{R}$ \\
\hline VCMP & 6150 & Maputo & - & - & $\mathrm{R}$ & $\mathrm{S}$ & $\mathrm{R}$ & $\mathrm{R}$ & $\mathrm{S}$ & $\mathrm{R}$ & $\mathrm{R}$ & $>\mathrm{R}$ \\
\hline VCMP & 6151 & Maputo & - & - & $\mathrm{R}$ & $\mathrm{R}$ & $\mathrm{R}$ & I & $\mathrm{S}$ & $\mathrm{R}$ & $\mathrm{R}$ & $>\mathrm{R}$ \\
\hline VCMP & 6223 & Maputo & + & 5 & $\mathrm{~S}$ & $\mathrm{~S}$ & $\mathrm{R}$ & $\mathrm{R}$ & $\mathrm{R}$ & $\mathrm{R}$ & $\mathrm{R}$ & $\mathrm{R}$ \\
\hline VCMP & 6242 & Maputo & - & - & $\mathrm{S}$ & $\mathrm{S}$ & I & $\mathrm{R}$ & $\mathrm{S}$ & $\mathrm{S}$ & I & $\mathrm{S}$ \\
\hline VCMP & 6246 & Maputo & - & - & $\mathrm{R}$ & $\mathrm{S}$ & $\mathrm{R}$ & $\mathrm{R}$ & $\mathrm{S}$ & $\mathrm{R}$ & $\mathrm{R}$ & $\mathrm{S}$ \\
\hline VCMP & 6416 & Maputo & - & - & $\mathrm{S}$ & I & I & $\mathrm{R}$ & $\mathrm{R}$ & $\mathrm{S}$ & I & $\mathrm{R}$ \\
\hline VCMP & 6421 & Maputo & - & - & $\mathrm{R}$ & I & I & I & $\mathrm{S}$ & $\mathrm{S}$ & I & $\mathrm{R}$ \\
\hline VCMP & 6425 & Maputo & + & 5 & $\mathrm{R}$ & I & $\mathrm{R}$ & I & $\mathrm{S}$ & $\mathrm{R}$ & I & $\mathrm{R}$ \\
\hline VCMP & 6427 & Maputo & - & - & $\mathrm{R}$ & I & $\mathrm{R}$ & $\mathrm{R}$ & $\mathrm{S}$ & $\mathrm{S}$ & $\mathrm{R}$ & $\mathrm{R}$ \\
\hline VCMP & 6429 & Maputo & - & - & $\mathrm{S}$ & $\mathrm{S}$ & I & I & $\mathrm{S}$ & $\mathrm{S}$ & I & I \\
\hline VCMP & 6430 & Maputo & - & - & $\mathrm{R}$ & $\mathrm{S}$ & I & $\mathrm{R}$ & $\mathrm{S}$ & $\mathrm{S}$ & I & $\mathrm{S}$ \\
\hline VCMP & 6455 & Maputo & - & - & $\mathrm{R}$ & $\mathrm{S}$ & I & $\mathrm{R}$ & $\mathrm{S}$ & $\mathrm{R}$ & $\mathrm{R}$ & $\mathrm{S}$ \\
\hline VCMP & 6466 & Maputo & - & - & $\mathrm{R}$ & $\mathrm{R}$ & I & $\mathrm{R}$ & $\mathrm{S}$ & $\mathrm{R}$ & $\mathrm{R}$ & $>\mathrm{R}$ \\
\hline VCMP & 6500 & Maputo & - & - & $\mathrm{R}$ & $\mathrm{S}$ & $\mathrm{R}$ & $\mathrm{R}$ & $\mathrm{R}$ & $\mathrm{R}$ & $\mathrm{R}$ & $>\mathrm{R}$ \\
\hline VCMP & 6505 & Maputo & + & 5 & $\mathrm{R}$ & $\mathrm{R}$ & I & I & $\mathrm{R}$ & $\mathrm{R}$ & $\mathrm{R}$ & $\mathrm{R}$ \\
\hline VCMP & 6508 & Maputo & - & - & $\mathrm{R}$ & I & I & I & $\mathrm{R}$ & $\mathrm{R}$ & I & $\mathrm{R}$ \\
\hline VCMP & 6517 & Maputo & - & - & $\mathrm{S}$ & $\mathrm{S}$ & I & $\mathrm{R}$ & $\mathrm{R}$ & $\mathrm{S}$ & $\mathrm{R}$ & I \\
\hline VCMP & 6520 & Maputo & - & - & $\mathrm{R}$ & $\mathrm{R}$ & $\mathrm{R}$ & I & $\mathrm{S}$ & $\mathrm{R}$ & $\mathrm{R}$ & $>\mathrm{R}$ \\
\hline
\end{tabular}


Table 1 (cont.)

\begin{tabular}{|c|c|c|c|c|c|c|c|c|c|c|c|c|}
\hline \multicolumn{3}{|c|}{ Isolate and place } & \multirow{2}{*}{$\frac{\text { CTX* }^{*}}{-}$} & \multirow{2}{*}{$\frac{\text { ERIC } \dagger}{-}$} & \multirow{2}{*}{$\frac{\mathrm{Amp}}{\mathrm{R}}$} & \multirow{2}{*}{$\frac{\text { Chl }}{\mathrm{R}}$} & \multirow{2}{*}{$\begin{array}{l}\text { Ery } \\
\text { I }\end{array}$} & \multirow{2}{*}{$\frac{\text { Kan }}{\mathrm{R}}$} & \multirow{2}{*}{$\begin{array}{l}\mathrm{Nal} \\
\mathrm{S}\end{array}$} & \multirow{2}{*}{$\frac{\text { Pen }}{\mathrm{R}}$} & \multirow{2}{*}{$\frac{\text { Rif }}{R}$} & \multirow{2}{*}{$\frac{\text { Tet }}{>\mathrm{R}}$} \\
\hline VCMP & 6551 & Maputo & & & & & & & & & & \\
\hline VCMP & 6552 & Maputo & + & 5 & $\mathrm{R}$ & $\mathrm{S}$ & I & $\mathrm{R}$ & $\mathrm{R}$ & $\mathrm{R}$ & $\mathrm{R}$ & $\mathrm{S}$ \\
\hline VCMP & 6553 & Maputo & - & - & - & I & I & - & $\mathrm{S}$ & - & - & $\mathrm{R}$ \\
\hline VCMP & 6998 & Maputo & + & 5 & $\mathrm{~S}$ & $\mathrm{~S}$ & I & I & S & $\mathrm{S}$ & I & $\mathrm{S}$ \\
\hline VCMP & 7036 & Maputo & - & 5 & $\mathrm{~S}$ & $\mathrm{~S}$ & I & I & S & $\mathrm{S}$ & I & I \\
\hline VCMP & 7038 & Maputo & + & 5 & $\mathrm{~S}$ & $\mathrm{~S}$ & I & I & $\mathrm{S}$ & $\mathrm{S}$ & I & $\mathrm{S}$ \\
\hline VCMP & 7042 & Maputo & - & 5 & - & $\mathrm{R}$ & I & - & $\mathrm{R}$ & - & - & $\mathrm{R}$ \\
\hline VCMP & 7048 & Maputo & + & 5 & $\mathrm{~S}$ & $\mathrm{~S}$ & I & I & $\mathrm{S}$ & $\mathrm{S}$ & I & I \\
\hline VCMP & 7053 & Maputo & - & 5 & $\mathrm{~S}$ & I & I & I & S & $\mathrm{S}$ & I & $\mathrm{R}$ \\
\hline VCMP & 7073 & Maputo & + & 5 & $\mathrm{~S}$ & $\mathrm{~S}$ & I & $\mathrm{R}$ & $\mathrm{R}$ & $\mathrm{S}$ & I & $\mathrm{R}$ \\
\hline VCMP & 9081 & Maputo & - & 5 & $\mathrm{~S}$ & $\mathrm{~S}$ & I & $\mathrm{R}$ & $\mathrm{R}$ & $\mathrm{S}$ & I & $\mathrm{R}$ \\
\hline VCMP & 10029 & Marracuene & + & 6 & $\mathrm{~S}$ & $\mathrm{~S}$ & I & I & $\mathrm{S}$ & $\mathrm{S}$ & I & $\mathrm{R}$ \\
\hline VCZB & 01 & Zambezia & + & 6 & $\mathrm{~S}$ & $\mathrm{~S}$ & I & - & S & - & - & $\mathrm{R}$ \\
\hline VCZB & 02 & Zambezia & - & - & $\mathrm{S}$ & $\mathrm{S}$ & $\mathrm{R}$ & - & S & $\mathrm{R}$ & - & $\mathrm{R}$ \\
\hline VCZB & 03 & Zambezia & - & - & $\mathrm{S}$ & I & I & - & S & $\mathrm{S}$ & - & $\mathrm{R}$ \\
\hline VCZB & 04 & Zambezia & - & - & - & $\mathrm{S}$ & - & - & S & - & - & $\mathrm{R}$ \\
\hline VCZB & 05 & Zambezia & - & - & $\mathrm{S}$ & $\mathrm{S}$ & I & - & $\mathrm{S}$ & $\mathrm{S}$ & - & $\mathrm{R}$ \\
\hline VCTT & 56 & Tete & + & 6 & $\mathrm{~S}$ & $\mathrm{~S}$ & I & - & $\mathrm{R}$ & $\mathrm{S}$ & - & $\mathrm{R}$ \\
\hline VCTT & 58 & Tete & - & - & $\mathrm{S}$ & $\mathrm{S}$ & I & - & $\mathrm{R}$ & $\mathrm{S}$ & - & $\mathrm{R}$ \\
\hline VCTT & 59 & Tete & - & - & $\mathrm{S}$ & $\mathrm{S}$ & I & - & $\mathrm{R}$ & $\mathrm{S}$ & - & $\mathrm{R}$ \\
\hline \multirow[t]{4}{*}{ Total } & 75 & 19 & 23 & 69 & 75 & 74 & 62 & 75 & 68 & 62 & 75 & \\
\hline & & & Total & $41 \mathrm{~S}$ & $51 \mathrm{~S}$ & $2 \mathrm{~S}$ & $0 \mathrm{~S}$ & $55 \mathrm{~S}$ & $48 \mathrm{~S}$ & $0 \mathrm{~S}$ & $12 \mathrm{~S}$ & \\
\hline & & & Total & $0 \mathrm{I}$ & $10 \mathrm{I}$ & $56 \mathrm{I}$ & $29 \mathrm{I}$ & $1 \mathrm{I}$ & $0 \mathrm{I}$ & $45 \mathrm{I}$ & $24 \mathrm{I}$ & \\
\hline & & & Total & $28 \mathrm{R}$ & $14 \mathrm{R}$ & $16 \mathrm{R}$ & $33 \mathrm{R}$ & $19 \mathrm{R}$ & $20 \mathrm{R}$ & $17 \mathrm{R}$ & $39 \mathrm{R}$ & \\
\hline
\end{tabular}

Starting from column 2: ${ }^{*}$ CTX, strains submitted to hybridization experiments to detect CTX element, — not determined; $\dagger$ ERIC, number of amplicons resulting by PCR amplification after $B g l$ I restriction of total DNA; columns 4-11: $\$$ S susceptible, I intermediate, R resistant MICs of the following drugs: Amp, ampicillin; Chl, chloramphenicol; Ery, erythromycin; Kan, kanamycin; Nal, nalidixic acid; Pen, penicillin; Rif, rifampicin; Tet, tetracycline ( $\geqslant 96 \mu \mathrm{g} / \mathrm{ml})$.

rifampicin, sulfonamide, tetracycline and trimethoprim. Breakpoint of susceptibility for each drug was defined according to Manual of Clinical Microbiology [24].

\section{PCR assay}

Preparation of chromosomal DNA for restriction enzyme digestions and PCR testing for toxin genes and ERIC sequences were performed as previously described $[12,13,25,26]$.

PCR detection of $t c p \mathrm{~A}, n a n \mathrm{H}, h l y \mathrm{~A}$ and $t o x \mathrm{R}$ pathogenic genes used the specifically designed primer pairs shown in Table 2. All 75 isolates were submitted to PCR detection of toxigenic and pathogenic factors.

Amplifications were performed in a reaction mixture of $25 \mu 1$ containing $10 \mathrm{~mm}$ Tris- $\mathrm{HCl}(\mathrm{pH} 8 \cdot 8)$, $50 \mathrm{~mm}$ potassium chloride, $1.5 \mathrm{~mm}$ magnesium chloride, $0 \cdot 1 \%$ Triton $\mathrm{X}-100,200 \mu \mathrm{M}$ of deoxynucleoside triphosphates and $100 \mathrm{pmol}$ of each primer. For amplification with TOXR1-TOXR2 and NANH1-
Table 2. Sequences of oligonucleotide primer pairs designed and used to detect various $\mathrm{V}$. cholerae virulence genes by PCR amplification

\begin{tabular}{lll}
\hline \hline Gene & Primer & Sequence \\
\hline tcpA & TCP1 & TGTTGTTACGCTGGCTCAGC \\
& TCP2 & AAGTTGGCACTTCCTGGTGC \\
hlyA & HLYA1 & AGCGTTCATTGCAATACGG \\
& HLYA2 & GGTCGAACGATTGAGTAGCG \\
toxR & TOXR1 & GAGATCATTCGATTAGGCAGC \\
& TOXR2 & TTATTCGCCACGACATTGG \\
nanH & NANH1 & GGTTGGTGCAAGGTATTGG \\
& NANH2 & TGAGGTTGAGCTCGGTATCC \\
\hline \hline
\end{tabular}

NANH2 primer pairs, $2.5 \mathrm{~mm}$ magnesium chloride was added. PCR conditions for all four primers included a pre-incubation step of $3 \mathrm{~min}$ at $94{ }^{\circ} \mathrm{C}$ and a final incubation for $5 \mathrm{~min}$ at $72{ }^{\circ} \mathrm{C}$. For TCP1-TCP2 and HLYA1-HLYA2 primers the PCR conditions included a middle step of 35 cycles of $1 \mathrm{~min}$ at $94^{\circ} \mathrm{C}$, $1 \mathrm{~min}$ at $57^{\circ} \mathrm{C}, 1 \mathrm{~min} 30 \mathrm{~s}$ at $72{ }^{\circ} \mathrm{C}$; but for TOXR $1-$ 
TOXR 2 and NANH1-NANH 2 primers, the middle step was 35 cycles of $1 \mathrm{~min}$ at $94^{\circ} \mathrm{C}, 1 \mathrm{~min}$ at $56^{\circ} \mathrm{C}$ and $1 \mathrm{~min} 30 \mathrm{~s}$ at $72{ }^{\circ} \mathrm{C}$.

PCR amplification of ERIC sequences was performed using DNA from 23 representative isolates (20 from Maputo city and 1 from each different province) and reference strains. Both undigested DNA and DNA digested by BglI (Amersham Life Science) and HindIII (Gibco-BRL) were used. Oligonucleotides for primer pairs were synthesized by Gene Assembler Plus (Pharmacia) as directed by the manufacturer, all PCR reactions contained 0.7 unit/reaction of Taq polymerase (DyNAzyme, Finnzyme Oy) and were carried out in a Perkin-Elmer Cetus DNA Thermal Cycler.

\section{DNA hybridization of CTX element}

To detect the copy number of the CTX genetic element, genomic DNA was digested by $X b a \mathrm{I}$ restriction enzyme (Gibco-BRL) that cleaves a unique site at the left end of the ctxA gene sequence (27). We studied a sample of 16 strains from Maputo and three from provinces affected by the 1997-8 epidemic and included three reference strains from Zimbabwe, Rwanda and Burundi. The molecular probe was the PCR ctxA amplicon from the $V$. cholerae O1 395 reference strain which spans a region to the right of the $X b a \mathrm{I}$ internal site. Restricted DNA was hybridized under low stringency conditions to the probe. The probe was labelled using fluorescein conjugated dUTPs and the random priming technique (BRL).

\section{Electrophoresis}

Detection of amplicons was made in $1 \%$ agarose (Biorad) gels in $40 \mathrm{~mm}$ Tris Acetate, $1 \mathrm{~mm}$ EDTA or in $1.8 \%$ (only for digested amplified DNA) agarose gels in $45 \mathrm{~mm}$ Tris Borate, $1 \mathrm{~mm}$ EDTA. Electrophoresis for Southern blot and DNA hybridization was made in $0.8 \%$ agarose gels in $40 \mathrm{~mm}$ Tris Acetate, 1 mм EDTA. Gels were stained by ethidium bromide. Marker VI (Boehringer-Mannheim, Germany) and 100 bp DNA Ladder (Life Technologies U.K.) were used as DNA mol. wt markers.

\section{RESULTS}

\section{Phenotypic characterization}

Biochemical and serological identification showed that all $V$. cholerae strains belonged to serotype $\mathrm{O} 1$
Ogawa, biotype El Tor. Susceptibility to each drug is reported in Table 1, columns 4-11. There was no conserved drug resistance profile among the epidemic strains. All isolates were susceptible (but three were intermediate) to ciprofloxacin out of 66 tested (not shown in Table 1). Interestingly only one strain (isolate VCMP 5807) was susceptible to sulfonamide and trimethoprim out of 72 tested (not shown in Table 1), as well as to kanamicin (29 intermediate), rifampicin (45 intermediate) and erythromycin (two susceptible and 53 intermediate). Only $16 \%$ of strains were susceptible to tetracycline and the MIC of six strains was more or equal to $96 \mu \mathrm{g} / \mathrm{ml}, 59 \%$ of isolates resulted susceptible to ampicillin, $68 \%$ to cloramphenicol and $70 \%$ to penicillin.

\section{Presence of virulence genes}

All 75 isolates were submitted to PCR amplification to detect virulence genes. All strains possessed the toxin genes and the other virulence factors investigated. No polymorphism was detected. The amplicons (sizes are expressed in bp) revealed by agarose gel electrophoresis resulted respectively: ace 284, zot 198 and ctxA 564, as previously reported [13] and tcpA 465, hlyA 509, toxR 395 and nanH 639 (Fig. 1). These four genes were detected by PCR for the first time and their sizes result consistent with gene bank sequences.

\section{CTX genetic element copy number}

The hybridization experiment to detect the copy number of the CTX genetic element in a representative group of 19 epidemic strains both from Mozambique (see Table 1, column 2) and from three other African countries, showed two $X b a \mathrm{I}$ restriction fragments respectively $9.4 \mathrm{~kb}$ and $17 \cdot 0 \mathrm{~kb}$ long (Fig. 2) indicating the presence of two CTX elements. Since the probe sequence is homologous to the region to the right of the $X b a \mathrm{I}$ site (unique inside each single CTX element), the duplication in tandem of CTX element doubles the number of $X b a \mathrm{I}$ sites and therefore the number of fragments hybridized by the $\operatorname{ct} x \mathrm{~A}$ probe.

\section{ERIC PCR fingerprints}

Analysis of ERIC PCR polymorphism of undigested DNA revealed a single fingerprint in the 23 isolates 

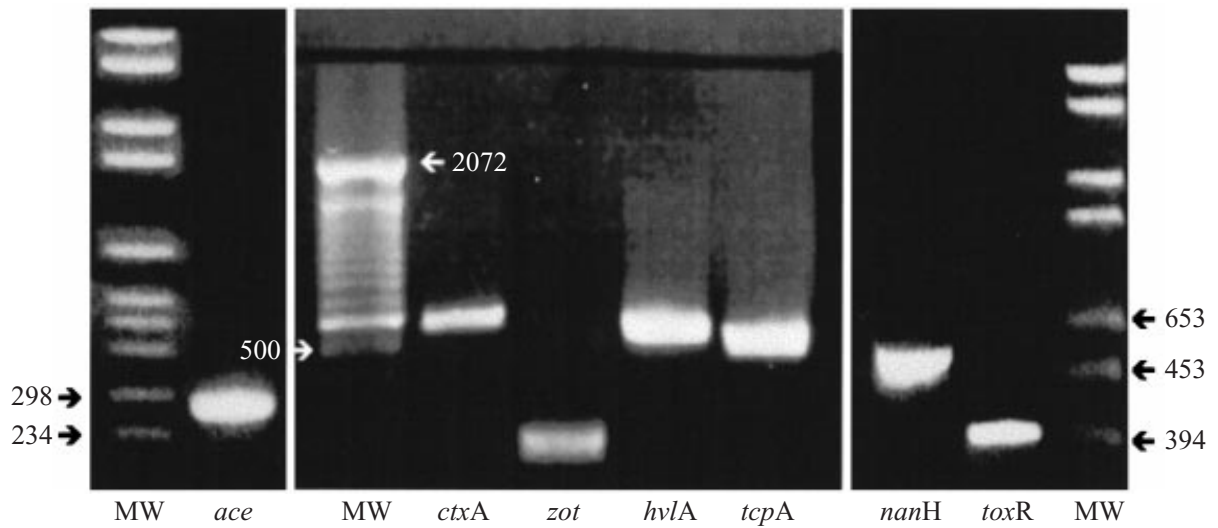

Fig. 1. Electrophoresis of agarose gels showing examples of specific amplicons synthesized by PCR amplification of $V$. cholerae virulence genes. Sizes resulted respectively (in bp): ace 284, zot 198, ctxA 564, tcpA 465, hlyA 509, toxR 395, nan $\mathrm{H}$ 639. MW, molecular weight standard, from the left respectively: marker VI, ladder 100 and marker VI.

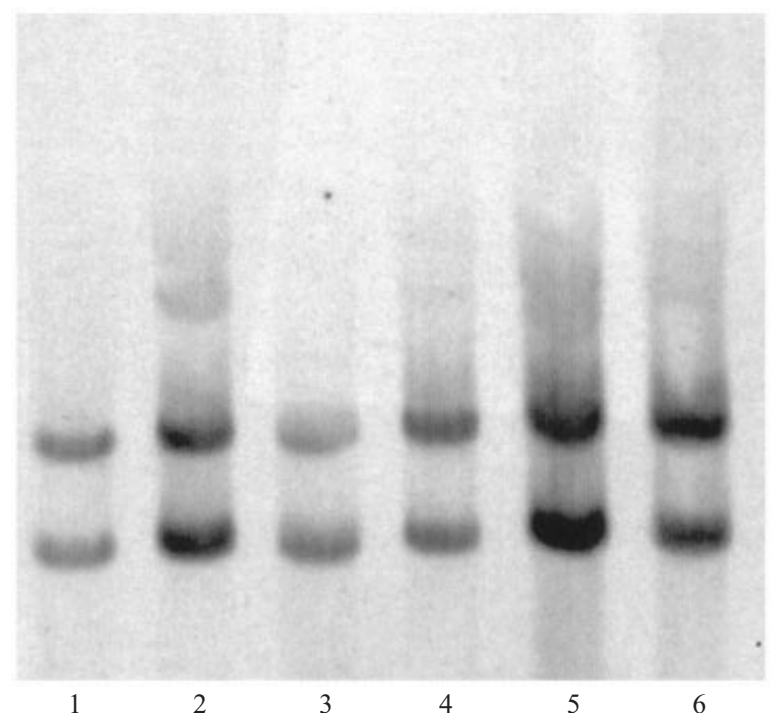

Fig. 2. Hybridization experiment showing two copies of CTX genetic element in 1997-8 Mozambican epidemic strains of $V$. cholerae. Lanes 1, 2: VCMP5556 and VCMP5806 isolates from Maputo city; lane 3: VCZB01 isolate from Zambézia; lanes 4-6: respectively Rwanda (CNRVC940183), Burundi (CNRVC940173) and Angola (VC663) isolates. Upper band and lower band correspond to fragment sizes of respectively $17 \cdot 0$ and $9 \cdot 4 \mathrm{~kb}$.

characterized by eight ERIC amplicons (Fig. 3A) : 3.0, $2 \cdot 6,1 \cdot 72,1 \cdot 44,1 \cdot 14,0 \cdot 89,0 \cdot 78,0.58 \mathrm{~kb}$ as previously reported [13]. Six African reference strains possessed the same pattern (data not shown).

In contrast, analysis of ERIC PCR polymorphism of $B g l I$ restricted DNA (Table 1, column 3) revealed a subtle difference among strains. All 20 isolates from Maputo city had a fingerprint of 5 amplicons (see Fig. 3B, lanes 1, 2, 6), 1.72, 1.44 and 0.89 ERIC amplicons are missing. Isolates from Marracuene district in

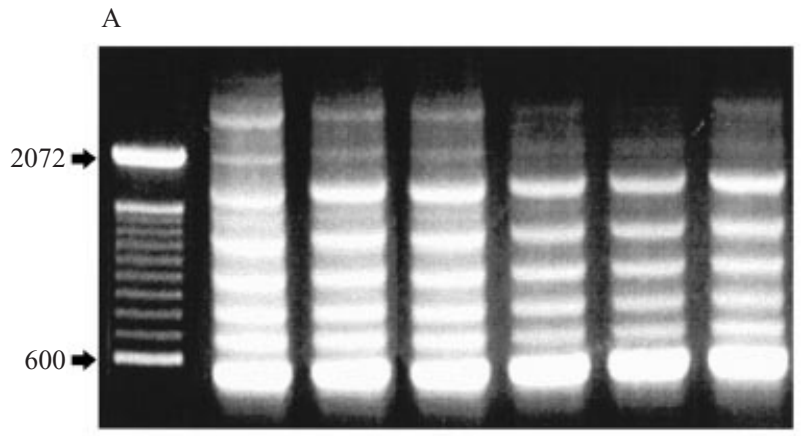

B

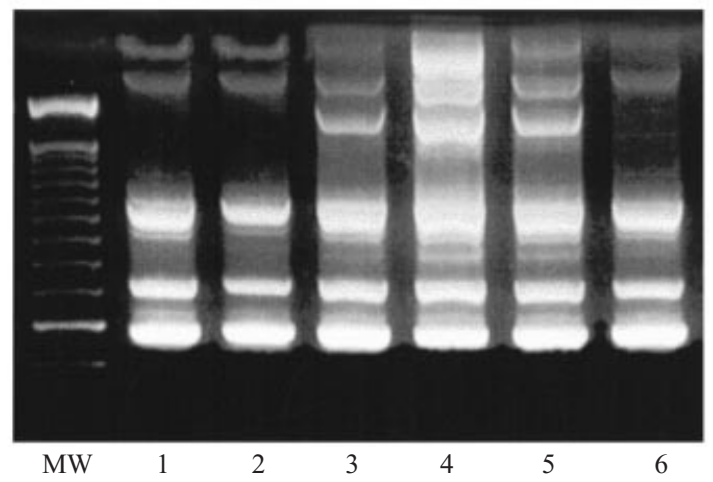

Fig. 3. Amplicon profiles of VCMP 5556, VCMP 5806, VCMP 10029, VCTT 56, VCZB 01 and VCMP 7073 Mozambican epidemic isolates of $V$. cholerae produced by PCR amplification of ERIC sequences. MW, molecular weight standard, ladder 100. Section A: undigested DNA, $1 \%$ agarose gel in Tris Acetate, showing ERIC profiles of eight amplicons: $3 \cdot 0,2 \cdot 6,1 \cdot 72,1 \cdot 44,1 \cdot 14,0 \cdot 89,0 \cdot 78,0 \cdot 58 \mathrm{~kb}$. Section B: digested DNA by $B g l \mathrm{I}$, in $1.8 \%$ agarose gel in Tris Borate. Lanes 1, 2, 6: DNA from 5556, 5806 and 7073 Maputo strains, 1.72, 1.44, and $0.89 \mathrm{~kb}$ ERIC amplicons are missing. Lanes 3-5, DNA from Marracuene (VCMP10029), Tete (VCTT 56) and Zambézia (VCZB01) strains, 1.44 and 0.89 ERIC amplicons are missing. 
Table 3. ERIC type distribution among African epidemic and reference strains of $\mathrm{V}$. cholerae

\begin{tabular}{lll}
\hline \hline Epidemic & Year & ERIC type \\
\hline Maputo, Mozambique* & $1997-8$ & $\mathrm{~B}$ \\
Provinces, Mozambique* & $1997-8$ & $\mathrm{~A}$ \\
Zimbabwe* & 1998 & $\mathrm{~A}$ \\
Swaziland* & 1998 & $\mathrm{~A}$ \\
Angola (13) & $1991-4$ & $\mathrm{~A}$ \\
Somalia (23) & $1985-6$ & $\mathrm{~A}$ \\
Rwanda & 1994 & $\mathrm{~A}$ \\
Burundi & 1994 & $\mathrm{~A}$ \\
Italy & 1994 & $\mathrm{~B}$ \\
India & 1992 & $\mathrm{~B}$ \\
India, V. cholerae O139 & 1992 & $\mathrm{~B}$ \\
India & 1995 & $\mathrm{~A}$ \\
India, V. cholerae O139 & 1998 & $\mathrm{~B}$ \\
\hline \hline
\end{tabular}

* Present study.

Maputo province, Tete and Zambézia provinces had a 6 amplicon fingerprint (Fig. 3B, lanes 3, 4, 5), since 1.44 and $0.89 \mathrm{~kb}$ ERIC amplicons are missing. This 6 amplicon fingerprint are shared by six African reference strains (data not shown). PCR of HindIII digested DNA also confirmed the existence of two ERIC types: the predominant African type A and a second type B in Maputo strains. Reference strains from Italy isolated in 1994 were ERIC type B. $V$. cholerae $\mathrm{O} 1$ and 0139 strains from India isolated in 1992 and 1998 were also ERIC type B, whereas the O1 strain isolated in 1995 was type A (see Table 3).

\section{DISCUSSION}

The 1997 epidemic was caused by $V$. cholerae O1 El Tor, serotype Ogawa, started in August (cold, dry season) in the poorest Maputo neighbourhoods and spread north to other provinces (in chronological order: Gaza, Inhambane, Manica, Sofala, Zambézia, Tete and Niassa). About 31500 cases (with 1.8\% mortality) were registered up to October 1998 and about 41000 (with $2.7 \%$ mortality) up to October 1999 (data obtained from Ministry of Health). According to the dynamics of geographic spread, the main mechanism of transmission appeared to be through contamination of water and food. The persistence of cholera with continuing sporadic cases may indicate a colonized environmental source as was described in 1991 [28].

Analysis of drug susceptibility data revealed a general resistance to sulfonamide and trimethoprim (cotrimoxazole) as well as a high resistance to tetracycline both in Maputo strains and in provincial isolates and the presence of multi-resistant strains. As the epidemic evolved there was an increasing proportion of multiple drug resistance (to at least five drugs including tetracycline). This trend may be explained by the exposure of $V$. cholerae strains to drugs utilized in clinical treatment and to genetic exchange with resistant bacterial flora.

The presence of conjugative plasmids harbouring a tetracycline resistance determinant associated with up to five resistance genes for other drugs (mainly kanamycin, ampicillin, penicillin, cloramphenicol and rifampicin), was found in epidemic $V$. cholerae $\mathrm{O} 1$ strains isolated in Somalia, Angola [13, 23, 29] and in other African countries. Conjugative plasmids and the presence of integrons [30] might explain the spread of highly resistant epidemic strains in Mozambique where cotrimoxazole and tetracyclines were widely used in therapy; these topics will be object of further investigations. This study also confirmed the ineffectiveness of doxycicline against $V$. cholerae to control the epidemic and the need for a moratorium on antibiotic therapy of cholera patients in Mozambique.

Although cholera is clearly important in Mozambique no previous molecular studies have been published that might give a better insight into these epidemics and the virulence of the strains.

We confirmed that cholera epidemic strains from Mozambique possess an intact set of virulence genes without apparent polymorphism. The hybridization experiment studying the genomic arrangement of CTX region, revealed two CTX genetic elements in all the strains tested, including reference strains.

To follow the epidemic spread of $V$. cholerae $\mathrm{O} 1$ in the country, we analysed ERIC sequence polymorphism as a molecular marker using Rivera's method of PCR amplification of whole DNA. A single ERIC type was obtained analysing undigested DNA of Mozambican strains irrespective of geographic origin or time of isolation during the epidemic. This ERIC fingerprint was also present in $V$. cholerae $\mathrm{O} 1$ strains belonging to other epidemics (Somalia 1983, Angola 1992, Rwanda and Burundi 1994, Zimbabwe and Swaziland 1998). This evidence lead to a modification of Rivera's method by the introduction of preliminary endonuclease restriction of DNA, before PCR testing. This procedure revealed two Mozambican ERIC types: the first in provincial isolates with six amplicons (type A), and the second in 
Maputo city with five amplicons (type B), missing the $1.72 \mathrm{~kb}$ amplicon. The first six amplicons ERIC type is also predominant in other African epidemic strains.

The Maputo ERIC type presumably originated by a rearrangement within the chromosome of the more widespread African strain. This genetic event inserted a sequence containing BgII and HindIII restriction sites inside the chromosomal region corresponding to the $1.72 \mathrm{~kb}$ ERIC amplicon, missing in Maputo strains. After digestion this region can no longer be amplified by PCR procedure. This short insertion is not detectable by PCR analysis of undigested DNA.

The origin of the Maputo outbreak is still unknown but Mozambican health authorities believed that the original strain was not indigenous since the outbreak began during the cold season, after a 5-year absence of the disease and with no evidence of endemic cases during the interval. The strains isolated in the provinces as the epidemic spread had a different ERIC type, the most common type in Africa. This might suggest that the Maputo ERIC type is not genetically stable and originated by a transient short insertional event. Another possible explanation is the introduction into Mozambique of two epidemic strains of different clonal origin, belonging to the two ERIC types. In this case provincial strains may have spread from central African refugees living in the north of Mozambique and Maputo strains might have been introduced by overseas travellers, since we also detected ERIC type B in Italy and India.

In conclusion, the phenotypic and genotypic characterization of Mozambican strains showed homogeneity among epidemic $V$. cholerae isolates. However, antibiotic resistance patterns were variable and Maputo isolates were genetically distinct from provincial isolates. ERIC PCR fingerprinting of restriction enzyme digested DNA greatly assisted the genetic tracking of the strains for epidemiological purposes.

\section{ACKNOWLEDGEMENTS}

We are grateful to Dr. A Barreto, Ministry of Health, for the data provided, Dr L. Da Costa for helpful discussion and Italian Embassy in Mozambique for its support. We thank Dr J. Melo and the technical staff of microbiology laboratory of Faculty of Medicine, Maputo, for technical assistance. This work was granted by University E. Mondlane and Ministry of Health, Mozambique; University Program DGCS - Ministry of Foreign Affairs, Ministry of
University Scientific Technologic Research, Cenci Bolognetti-Ist. Pasteur Foundation and Regione Autonoma Sardegna LR 19/96 Italy.

\section{REFERENCES}

1 Aly FAR, Machatine GJ, Paulo D. Epidemia de colera na provincia de Maputo Novembro 1991-Junho 1992. Rev Med Moçambique 1993; 4: 2-4.

2 Folgosa E, Valdivia JA, Hung M. Colera na cidade e provincia de Maputo: resultados laboratoriais das amostras analisadas no periodo de Junho 1991 a Junho 1992. Rev Med Moçambique 1994; 5: 9-12.

3 Keasler SP, Hall RH. Detecting and biotyping Vibrio cholerae $\mathrm{O} 1$ with multiplex polymerase chain reaction. Lancet 1993; 341: 1661.

4 Van Belkum F. DNA fingerprinting of medically important microorganism by use of PCR. Clin Microbiol Rev 1994; 7: 174-84.

5 Kostman JR, Edlind TD, LiPuma JJ, Stull TL. Molecular epidemiology of Pseudomonas cepacia determined by polymerase chain reaction ribotyping. $\mathbf{J}$ Clin Microbiol 1992; 30: 2084-7.

6 Popovic T, Bopp C, Olsvik Ø, Wachsmuth K. Epidemiologic application of a standardized ribotype scheme for Vibrio cholerae O1. J Clin Microbiol 1993; 31 : 2474-82.

7 Faruque SM, Alim ARMA, Roy SK, Khan F, et al. Molecular analysis of rRNA and cholera toxin genes carried by the new epidemic strain of toxigenic Vibrio cholerae O139 synonym Bengal. J Clin Microbiol 1994; 32: 1050-3.

8 Kostman JR, Alden MB, Mair M, Edlind TD, LiPuma JJ, Stull TL. A universal approach to bacterial molecular epidemiology by polymerase chain reaction ribotyping. J Infect Dis 1995; 171 : 204-8.

9 Clark CA, Purins L, Kaewrakon P, Manning PA. VCR repetitive sequence elements in the Vibrio cholerae chromosome constitute a mega-integron. Mol Microbiol 1997; 26: 1137-43.

10 Radu S, Ho YK, Lihan S. et al. Molecular characterization of Vibrio cholerae $\mathrm{O} 1$ and non-O1 from human and environmental sources in Malaysia. Epidemiol Infect 1999; 123: 225-32.

11 Liu PY, Shi Z, Lau Y, et al. Comparison of different PCR approaches for characterization of Burkholderia (Pseudomonas) cepacia isolates. J Clin Microbiol 1995; 33: 3304-7.

12 Rivera GI, Chowdhury MAR, Huq A, Jacobs D, Martins MT, Colwell R. Enterobacterial repetitive intergenic consensus sequences and the PCR to generate fingerprints of genomic DNAs from Vibrio cholerae O1, O139, and non-O1 strains. Appl Environ Microbiol 1995; 61 : 2898-904.

13 Colombo MM, Mastrandrea S, Leite F, et al. Tracking of clinical and environmental Vibrio cholerae $\mathrm{O} 1$ strains by combined analysis of the presence of toxin cassette, plasmid content and ERIC PCR. FEMS Imm Med Microb 1997; 19: 33-45. 
14 Shangkuan YH, Tsao CM, Lin HC. Comparison of Vibrio cholerae $\mathrm{O} 1$ isolates by polymerase chain reaction fingerprinting and ribotyping. J Med Microbiol 1997; 46: $941-8$.

15 Baudry B, Fasano A, Ketley J, Kaper JB. Cloning of a Gene (zot) encoding a new toxin produced by Vibrio cholerae. Infect Immunol 1992; 60: 428-34.

16 Fasano A, Baudry B, Pumplin DW, et al. Vibrio cholerae produces a second enterotoxin, which affects intestinal tight junctions. Proc Natl Acad Sci USA 1991; 88: 5242-6.

17 Trucksis M, Galen JE, Michalski J, Fasano A, Kaper JB. Accessory cholera enterotoxin (Ace), the third member of a Vibrio cholerae virulence cassette. Proc Natl Acad Sci USA 1993; 90: 5267-71.

18 Pearson GDN, Woods A, Chiang SL, Mekalanos JJ. CTX genetic element encodes a site-specific recombination system and an intestinal colonization factor. Proc Natl Acad Sci USA 1993 ; 90 : 3750-4.

19 Waldor MK, Mekalanos JJ. Lysogenic conversion by filamentous phage encoding cholera toxin. Science 1996; 272: 1910-4.

20 Hase CC, Mekalanos JJ. TcpP protein is a positive regulator of virulence gene expression in Vibrio cholerae. Proc Natl Acad Sci USA 1998; 95: 730-4.

21 Ghosh C, Nandy RK, Dasgupta SK, Nair GB, Hall $\mathrm{RH}$, Ghose AC. A search for cholera toxin (CT), toxin coregulated pilus (TCP), the regulatory element Tox R and other virulence factors in non-O1/non-O139 Vibrio cholerae. Microb Pathogen 1997; 22: 199-208.

22 Kaper JB, Fasano A, Trucksis. Toxins of Vibrio cholerae. In: Wachsmuth IK, Blake PA, Olsvik Ø, eds. Vibrio cholerae and cholera. Washington, D.C.: ASM Press, 1994: 157-60.
23 Coppo A, Colombo MM, Pazzani C, et al. Vibrio cholerae in the Horn of Africa: epidemiology, plasmids, tetracycline resistance gene amplification, and comparison between $\mathrm{O} 1$ and non-O1 strains. Am J Trop Med Hyg 1995; 53: 351-9.

24 Murray PR, Baron EJ, Pfaller MA, Tenover FC, Yolken RH. Manual of clinical microbiology, 6th ed. N. W.: ASM, 1995.

25 Ausbel FM, Brent R, Kingston RE, et al. Current protocols in molecular biology. 2: 4.1-5. New York: Greene Publishing Associates and Wiley Interscience, 1990.

26 Fields PI, Popovic T, Wachsmuth K, Olvik Ø. Use of polymerase chain reaction for detection of toxigenic Vibrio cholerae $\mathrm{O} 1$ strains from the Latin American cholera epidemic. J Clin Microbiol 1992; 30: 2118-21.

27 Basu A, Mukhopadhyay AK, Sharma C, et al. Heterogeneity in the organization of the CTX genetic element in strains of Vibrio cholerae O139 Bengal isolated from Calcutta, India and Dhaka, Bangladesh and its possible link to the dissimilar incidence of O139 cholera in the two locales. Microb Pathogen 1998; 24: 175-83.

28 Collins AE. The geography of cholera. In: Drasar BS, Forrest BD, eds. Cholera and the ecology of Vibrio cholerae. London: Chapman \& Hall, 1996: 263-6.

29 Colombo MM, Francisco M, Ferreira BD, Rubino S, Cappuccinelli $\mathrm{P}$. The early stage of the recurrent cholera epidemic in Luanda, Angola Eur J Epid 1993; 9: 563-5.

30 Falbo V, Carattoli A, Tosini F, Pezzella C, Dionisi AM, Luzzi I. Antibiotic resistance conferred by a conjugative plasmid and a class I integron in Vibrio cholerae $\mathrm{O} 1 \mathrm{El}$ Tor strains isolated in Albania and Italy. Antimicrob Agents Chemother 1999; 43: 693-6. 